\title{
BMJ Open Exploring general practitioners' views and experiences on suicide risk assessment and management of young people in primary care: a qualitative study in the UK
}

Maria Michail, Lynda Tait

To cite: Michail M, Tait L. Exploring general practitioners' views and experiences on suicide risk assessment and management of young people in primary care: a qualitative study in the UK. BMJ Open 2016;6: e009654. doi:10.1136/ bmjopen-2015-009654

- Prepublication history and additional material is available. To view please visit the journal (http://dx.doi.org/ 10.1136/bmjopen-2015009654).

Received 7 August 2015 Revised 14 December 2015 Accepted 15 December 2015

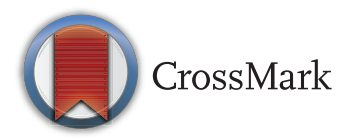

School of Health Sciences, Jubilee Campus, University of Nottingham, Nottingham, UK

Correspondence to Dr Maria Michail; maria. michail@nottingham.ac.uk

\section{ABSTRACT}

Objective: To explore general practitioner (GP) views and experiences of assessing, communicating with and managing suicidal young people with the aim of coproducing an educational intervention on youth suicide prevention tailored to GPs' perceived needs.

Design: Qualitative focus group study using framework analysis.

Setting: 5 inner city general practices in Nottingham. Participants: 28 GPs took part (9 males) with mean age of 37 years. The median number of years of professional experience was 13 . Participants were recruited through convenience sampling based on accessibility, interest in the study and willingness to participate.

Results: 3 themes emerged from the data in relation to GP's attitudes and beliefs towards suicide; the challenges GPs experience when it comes to the assessment and management of suicide risk in young people; and optimal ways of addressing some of these challenges through the provision of specialist

education and training targeting GPs' knowledge and clinical skills in this field.

Conclusions: The findings revealed wide variations in the understanding and operationalisation of risk among GPs, which has subsequent implications to how GPs perceive risk should be assessed. GP education on suicide risk assessment and management in youth should promote a holistic understanding and assessment of risk and its individual, social and contextual influences.

\section{BACKGROUND}

Suicide is the second leading cause of death in young people aged 15-29 worldwide. $^{1}$ Parasuicide phenomena such as previous suicide attempts, suicidal ideation and deliberate self-harm are even higher among youth and are associated with completed suicide. ${ }^{2-4}$ Self-harm in particular is among the strongest predictors of suicide with $80 \%$ of young

\section{Strengths and limitations of this study.}

- The study was presented in line with the Consolidated Criteria for Reporting Qualitative Research (COREQ) ensuring the explicit and comprehensive reporting of all study elements.

- Convenience sampling based on accessibility, interest in the study and willingness to participate might have led to over-representation or under-representation of particular groups of general practitioners (GPS) within the sample. Recruiting GPs with an interest in mental health might have led to a bias towards increased confidence in managing youth mental health issues including suicide risk.

- Despite the richness of data collected, pragmatic constraints such as a time-limited space to conduct the focus groups might have influenced the depth of discussion among the participants on such a complex issue as youth suicide prevention.

people who die by suicide having self-harmed in the past year. ${ }^{5}$ Youth suicide prevention is a global public health target. ${ }^{1}$ In England, the national strategy for the prevention of suicide $^{6}$ has highlighted the importance of the early identification and management of at-risk young people.

Primary care presents an excellent opportunity for early identification and assessment of suicide risk as evidence shows increased rates of contact with general practitioners (GPs) by those completing suicide, including young adults, 1-3 months before their death. ${ }^{7}$ GPs are often the first point of contact for people in distress ${ }^{9}$ and concerning mental health problems, young people are more likely to seek professional help from a GP. ${ }^{10}$ Moreover, we know that $70-90 \%$ of young people visit their GP at least once a year, ${ }^{11-13}$ with an estimated 
average of 2-3 times/year. ${ }^{14}$ GPs therefore can play an important role in the screening and identification of at-risk young people.

Suicide risk assessment, however, is an area that most GPs find particularly challenging. Time limited consultations coupled with lack of specialist clinical skills and inadequate mental health training have been identified by GPs as significant barriers to the assessment and management of suicidal presentations. ${ }^{15}$ Risk factors such as depression are not systematically detected and managed by GPs, ${ }^{16-19}$ while recent findings indicate that the prevalence and manifestation of self-harm in young people is underestimated by GPs. ${ }^{20}$ Younes et $a l^{16}$ showed that, compared with older adults, young adults were offered significantly fewer interventions by their GPs in the 3 months preceding their attempted suicide including fewer referrals to specialist mental health services. Communication difficulties reported by GPs and young people during consultations for mental health problems could hamper engagement as well as the development of a trusting doctor-patient relationship. ${ }^{21}$

Providing education and training to GPs in identifying, assessing and managing suicidal behaviour in young people has been highlighted as an important suicide prevention strategy. ${ }^{122} 23$ A systematic review conducted by the authors ${ }^{24}$ has highlighted the lack of evidence-based educational interventions for GPs targeting suicide risk in youth aged 14-25. The need for specialised provider training and continuing medical education in this field could have the potential to enhance GP knowledge and skills in the assessment and management of youth suicide risk, improving health outcomes for young people.

The aim of this study was to explore GPs' views and experiences of assessing, communicating with, and managing suicidal young people aged 14-25 that would inform the development of an educational intervention for GPs on youth suicide prevention tailored to their perceived needs and feasible to be delivered in primary care.

\section{METHODS}

Study design

This was a qualitative focus group study conducted in Nottingham City, UK, between October and April 2015. The study received ethical approval by NRES Committee West Midlands-Coventry \& Warwickshire (14/WM/ 1100). The study is presented in line with the Consolidated Criteria for Reporting Qualitative Research $(\text { COREQ })^{25}$ (see online supplement file A).

\section{Research team and reflexivity}

Focus groups with GPs were conducted by a trained interviewer (MM). The interviewer was unknown to participants prior to the start of study. Participants were made aware of the study goals and professional status of the interviewer.

\section{Sample}

All GPs from the 62 general practices in Nottingham City, serving a population of 325000 , were eligible to participate in the study. GPs were recruited by convenience sampling based on accessibility, interest in the study and willingness to participate. The study team initially approached the four GP cluster leads in Nottingham City Clinical Commissioning Group (CCG) to facilitate recruitment. An email was sent by the GP cluster leads to all GPs in Nottingham City practices informing them about the study. Recruitment was also facilitated by the Clinical Research Network (CRN): East Midlands. GP practices expressing an interest in the study approached the study team via their practice manager to book an appointment for a focus group.

\section{Data collection and analysis}

Four practice-based focus groups and one in-depth interview (single-handed practice) were conducted, with responses indicating that saturation had been achieved. Focus groups were based on a topic guide (see online supplementary file B) to ensure all key areas were covered, informed by previous literature, and piloted in an initial focus group. The interviewer initiated the interview by introducing herself, explaining the purpose of the study and the procedure to follow. The interviewer reminded GPs of issues around confidentiality and anonymity. GPs were invited to speak freely, with additional probes and prompts as required. Focus groups lasted approximately $45 \mathrm{~min}$ and took place during lunchtime practice meetings or when practices were closed to the public. Written consent was obtained from all GPs participating in the focus groups prior to each focus group taking place. Focus groups were digitally recorded with permission from the GPs. Transcripts were fully transcribed verbatim, and edited to remove names to preserve participant anonymity. The interviewer kept field notes in the form of memos. The focus group data were analysed using framework analysis, ${ }^{2627}$ a structured and rigorous process of theme-based analysis through the development of charts. ${ }^{28}$ Adopting framework analysis allowed the authors to work independently and then collaboratively to develop, review and refine themes and subthemes through an iterative process. No software was used for data analysis.

\section{FINDINGS}

Twenty-eight GPs participated (FG1=7 GPs; FG2=7 GPs; FG3=7 GPs; FG4=6 GPs; 1 in-depth interview), 9 of whom were males; 15 were females. Four participants did not record their gender. The demographic characteristics of the sample are presented in table 1 .

Each of the three key themes that emerged from the data is presented below and supported by illustrative quotations from the transcripts.

\section{CHALLENGES IN THE ASSESSMENT AND MANAGEMENT OF SUICIDE RISK IN YOUNG PEOPLE Lack of specialist knowledge and clinical skills}

There was consensus among GPs about the lack of learning modules on youth suicide prevention. Although GPs 
Table 1 Characteristics of study participants

\begin{tabular}{|c|c|c|c|c|c|}
\hline No & Age & Gender & $\begin{array}{l}\text { Years of } \\
\text { professional } \\
\text { experience }\end{array}$ & $\begin{array}{l}\text { Practice } \\
\text { size }\end{array}$ & $\begin{array}{l}\text { Attendance } \\
\text { of recent } \\
\text { training }\end{array}$ \\
\hline 1 & 46 & 1 & 23 & 4 & 1 \\
\hline 2 & 36 & 1 & 13 & 4 & 1 \\
\hline 3 & 41 & 0 & 19 & 4 & 1 \\
\hline 4 & 34 & 0 & 11 & 4 & 0 \\
\hline 5 & 34 & 1 & 9 & 4 & 1 \\
\hline 6 & 32 & 1 & 10 & 4 & 1 \\
\hline 7 & 39 & 1 & 16 & 4 & 1 \\
\hline 8 & 37 & 999 & 13 & 3 & 999 \\
\hline 9 & 37 & 999 & 13 & 3 & 999 \\
\hline 10 & 37 & 999 & 13 & 3 & 999 \\
\hline 11 & 37 & 1 & 13 & 3 & 1 \\
\hline 12 & 34 & 1 & 7 & 3 & 1 \\
\hline 13 & 47 & 1 & 24 & 3 & 1 \\
\hline 14 & 37 & 0 & 8 & 3 & 1 \\
\hline 15 & 37 & 0 & 13 & 3 & 1 \\
\hline 16 & 25 & 0 & 1.6 & 3 & 1 \\
\hline 17 & 37 & 0 & 30 & 3 & 1 \\
\hline 18 & 37 & 0 & 40 & 3 & 1 \\
\hline 19 & 34 & 1 & 10 & 4 & 1 \\
\hline 20 & 37 & 0 & 28 & 4 & 1 \\
\hline 21 & 26 & 1 & 1.6 & 4 & 1 \\
\hline 22 & 55 & 0 & 31 & 2 & 0 \\
\hline 23 & 52 & 1 & 22 & 4 & 1 \\
\hline 24 & 27 & 1 & 8 & 3 & 0 \\
\hline 25 & 38 & 1 & 13 & 4 & 1 \\
\hline 26 & 40 & 1 & 15 & 1 & 1 \\
\hline 27 & 27 & 1 & 2 & 3 & 1 \\
\hline 28 & 37 & 999 & 13 & 3 & 999 \\
\hline \multicolumn{6}{|c|}{$\begin{array}{l}\text { 0, male; } 1 \text {, female; practice size: } 1,<3000 ; 2,3000-5999 ; \\
3,6000-8999 ; 4,>9000 \text {. } \\
999, \text { missing value. } \\
\text { Training: } 0 \text {, no; } 1 \text {, yes. }\end{array}$} \\
\hline
\end{tabular}

felt relatively confident in dealing with general mental health problems, they felt ill-equipped to assess and manage effectively suicide risk in young people. Uncertainty about specific youth suicide predictors as well as a weighting approach to accurately identify risk emerged from GPs' narratives.

[...] family history, writing about death, expressing hopelessness, planning (...) poverty, I mean, I think I probably (I would tick) to most of those, actually. So, I suppose, what I don't have a sense of what weighting to give, you know, are there five there that, you know, if you tick yes to all five then actually you're in the red zone, I don't really... (GPF2 FG1)

Equally challenging for GPs was the accurate recognition of warning signs and how to distinguish between signs indicating imminent suicide risk from behavioural and affective changes that form part of 'normal adolescence'. Linked to this was a general uncertainty among GPs about distinguishing between what they called 'truly suicidal behaviour' and a 'cry for help' in young people.
This meant they often felt uncertain about management options, that is, when to make a referral to secondary mental health services and how to appropriately respond to a 'cry for help' particularly when it comes to young people engaging in self-harming behaviour.

It's very difficult to find who's really suicidal because, as I said, we do, most of the time, they will not come here and say I'm suicidal. Yes, there are a few of them, again, I don't know how much (...) they're young people but they're $(\ldots)$ the one if they are depressed, they do use it as, you know, this cry for help, whether they're actually, most of the time it happens, they're actually not going to do anything about it, and, but they just trying to get some attention, or some help at the time. (GP FG4)

...Which ones should we arrange further review for or the crisis team etc? Who is safe for us to keep and monitor in whatever time period that is and who needs actually to be seen now by somebody else? (GPM1 FG1)

GPs highlighted the importance of open communication and direct questioning about suicide as a way of facilitating disclosure and combating suicide-related stigma. However, the majority of GPs expressed concerns about the optimal way of communicating openly with young people during consultations. Building rapport and finding the most appropriate way of asking sensitive questions, particularly when dealing with young people who were not forthcoming, had very complex presentations, or were accompanied by a parent, made the risk assessment process very challenging for GPs.

(...) but with the younger people, as I said, probably, I think it is difficult, they wouldn't be that much forthcoming which is sometimes (...), sometimes they are good as well, don't say that everybody is the same. But sometimes it can be a bit difficult as well, or they will say, Oh, we don't want to discuss (...) (detail) if it brings the things back, because mostly (...) people, there's something, you know, more deep-rooted or they don't want to talk against, if there's something in the family, if they've come in with the family, that sometimes can be a bit difficult. (GPF FG4)

An additional challenge that some GPs faced was balancing confidentiality and disclosure of risk when working with at-risk young people, particularly those under 16. GPs were concerned that the need for breaching confidentiality could potentially alienate young people and impede a trusting doctor-patient relationship. Reflecting on their ability to strike a balance, GPs acknowledged certain skill deficits in how to appropriately phrase questions and communicate the need for parental involvement where they felt it was necessary:

...the times that I've often said, would you like me to speak to your mum or dad, I am quite concerned about you, they always, I don't know what I say, but they always say No thanks, I don't want you to talk to them...I'd 
quite like to be able to know what I'm saying wrong. (GPf6 FG1)

\section{Patient-related barriers}

GPs talked extensively about patient-related barriers to suicide prevention with lack of help seeking and an unwillingness to reveal the true extent of distress and suicidal intentions identified as the most significant issues. GPs also believed that young people who are 'truly' suicidal and have a dedicated plan would most likely not seek help or share their plans with their GP.

(...) the majority of them that had gone out and killed themselves hadn't come in to tell us about it first. We hadn't seen one of them for two years, and one I'd seen with her daughter but she'd never presented on her own...So they don't, they don't come in. If they're going to do it, they're going to do it. (GPR F2)

GPs also identified that the involvement of caregivers in consultations could sometimes prove to be counterproductive. GPs acknowledged the importance of involving caregivers in consultations and encouraged young people to reach out to friends and family for help. However, they expressed concerns that in some cases such involvement could hinder an open communication with young people by preventing them from revealing the true extent of their distress, symptoms or suicidal ideation:

(...) if they've come in with the family, that sometimes can be a bit difficult. I do always offer if I think that, you know, the mum is here, dad is here, and that, and they're not coming up with anything, I do offer them, would you like me to ask mum or dad to go out? Sometimes, they do, you know, and we do it, but sometimes probably they know that (...) if they go out, they will know that probably they are going to tell me anything so it can be, I think it all depends on individual really... (GP FG4)

\section{Organisational barriers}

Difficulties in accessing secondary mental health services, dysfunctional referral pathways and lack of communication with mental health providers were identified by GPs as significant obstacles to the effective management of suicidal young people.

I think that's something that a lot of GPs empathise with. [laughter] The sort of the long waiting times and those phone calls to the crisis, (...) and obviously, although we do whatever we can but they are (referring to crisis team) the specialism, isn't it, psychiatry? So then we want some specialist help sometimes, there can be a bit of a resistant (there), you know, taking the patient over. (GP FG4)

What the above account reveals is GPs' frustration with having to 'force' crisis teams to consider patients they felt were high priority. This alludes to poor communication with crisis teams primarily due to the lack of clarity about the severity levels of risk required for referral. The following account is indicative of poor liaison between primary care and mental health professionals.

But some day, we do find it a bit difficult for the crisis team to take, Oh, have you done that? you've seen the patient yourself? and it looks like, come on, I'm telling you what I know, and it can be sometimes a bit of, you know, battle of, please take it, (...) we don't want to take it. (GP FG4)

Practice constraints such as time limited consultations and a heavy workload were perceived by GPs to be significant barriers to conducting a thorough suicide risk assessment. This often left GPs feeling frustrated and concerned that they only get to see 'a snapshot' of young people's lives in a $10 \mathrm{~min}$ consultation which makes it is very difficult to form an accurate picture of the social context and circumstances to reliably assess risk. GPs reported that very often assessment of risk and associated management is based on clinical judgement or 'gut feeling' with all GPs emphasising the need for regular review of at-risk young people along with a 'watch and wait' approach for depression. One GP said:

I think the other thing which is difficult is most of the time, especially in the younger people, it is very more circumstantial, I don't know, broke up with a boyfriend, you know, new relation, bit of a stress in the family situation, maybe some abuse, and the problem is only come and see us for the ten minutes, so it can sometimes be quite difficult and most of the management is usually done, the community counselling, so this kind of an engagement to see them that regularly, can be sometimes quite a bit challenging really. (FG4)

\section{ATTITUDES AND BELIEFS OF GPS}

The majority of GPs believed suicide is very difficult to predict and therefore unpreventable, which left them feeling powerless to influence the course of events once a young person has decided to end their life. One GP questioned:

Maybe that's that concept of actually, is a suicide preventable? That's, maybe that's, you know, a negative aspect from my point of view, thinking perhaps they aren't preventable when actually they are, aren't they? (FG2R)

GPs revealed that they view young people as impulsive and unpredictable, which could lead to unintended completed suicides that could make the task of distinguishing between serious suicidal intent and 'attention seeking' very challenging. Self-harm was frequently seen by GPs as a way young people seek attention from family or healthcare professionals. They described young people as not always transparent about the extent of their suicidal intentions and plans leading to either minimisation or denial of their difficulties. This made managing risk very challenging for GPs, with some acknowledging an element of 'heartsink' in how they perceived young people: 
Yeah, I'd say, generally, any person with a depression is [laughter] a bit of a heartsink. [laughter] Because I think, as I said, my personal experience, you know, that this consultation is not going to finish in ten minutes, okay. So that can be a bit of a difficulty. But again, I think it depends on, not every patient will be a heartsink, sometimes they are easy to manage, they are happy whatever you suggest, they will do, okay? But some of them which are probably, (...) they are not very forthcoming, or whether, they are wanting some kind of a magical quick resolution which is not possible in depression, isn't it, (...) yeah, I think it depends on patient to patient really. (FG4)

\section{WAYS OF ADDRESSING CHALLENGES IN THE ASSESSMENT AND MANAGEMENT OF YOUTH SUICIDE RISK IN PRIMARY CARE \\ Provision of specialist education}

GPs acknowledged the need for specialist education to improve their knowledge and clinical skills in the assessment and management of suicide risk in young people. There was general consensus that further education on youth-specific suicide predictors, including those related to the social context (eg, bullying) of young people would be valuable for GPs. Having a list of questions to use during consultations followed by training on how to ask these questions would alleviate GPs' anxiety related to professional performance and would provide a benchmark for good practice in the assessment and management of suicide risk in primary care. Training on how to effectively involve family members in consultations without jeopardising doctor-patient confidentiality was also highlighted.

In terms of clinical skills, GPs emphasised the need for specialist training on communication and managing challenging consultations with young people. They proposed the involvement of young people in the delivery of such training to obtain their views and expectations from a GP consultation.

(...) comm- communication skills for the younger, younger, adolescents...really. Because they switch off, when you ask them questions (...). Yes, [agreeing with receiving training on how to ask sensitive questions], but also, what to do when you reach a blank wall. (GPRM6 FG3)

Enhancing GPs' therapeutic skills was a recurrent theme emerging from the data. GPs acknowledged that consultations are primarily focused on assessment and pharmacological management with very little therapeutic input. Providing GPs with the necessary clinical skills to deliver brief psychological interventions and counselling to manage distress was suggested to significantly facilitate consultations with young people by enhancing engagement and management options.

Something that I did a few years ago was a workshop and some brief interventions in psychology and it was quite helpful...it was quite good. I'd like a refresher in that really because we, do a lot of questions, me asking questions, we go over a lot of things which, I suppose, to them, it is, for us, it's educational for them, it may be going over more of the negativity that's brought them to us (...) in the first place, and like you say, I'd like to be a then, to be able to take that, and be able to offer something back which is, which has to be (necessarily) fairly brief, but still (...) be effective as possible. (FG1 GPm5)

\section{Educational content and implementation}

GPs expressed their views on optimal, feasible and acceptable ways of developing and delivering an educational intervention to support them in the identification and management of suicide risk in young people. Small, face-to-face group training in practice during clinical meetings or with multiple practices and a cross-section of GPs' length of experience was proposed. Online resources were proposed as a practical and cost-effective way of supplementing the main training session. GPs highlighted the importance of adapting the training for different learning styles and years of experience.

I think online are a good back-up for people who are time-pressed but I don't think there's anything to beat actually meeting in small groups, and particularly with, not just your own practice, I think it's really useful to, at cross (practice) event for, like, you know, for like, this focus group, but, you know, groups of say, up to fifteen or so, where, you know, there's enough conversation going without people feeling too intimidated but also, supported. And a cross section of experienced and younger GPs as well, because we do learn from each other. (FG3 RF1)

For the successful delivery and implementation of the intervention, GPs emphasised the role of a professional with a good understanding of the role of GPs and experience in conducting risk assessments. There was consensus that a GP with youth mental health expertise would be the most suitable trainer supported by a representative from the mental health provider (eg, CAMHS or crisis teams). A short film or video clip on young people's experiences and views on how to improve GP service provision was supported by most GPs.

I think that [referring to the involvement of young people in the delivery of training] would be really good...or even (...) what we do is what we think is right from our point of view, but we may be doing something completely, ignoring something which is important for these young people. I think that would be very good. To know what they're [thinking]. (FG4)

\section{Provision of suicide risk assessment tool}

As a way of addressing communication challenges between GPs and the mental health provider, some GPs supported the use of a mutually agreed validated suicide risk assessment tool across primary and secondary care that would facilitate the accurate identification of those 
at-risk and inform decision-making about their management.

Yeah, the other thing is, if you had a threshold or a score, it would help us convey very simply to someone like the crisis team the level of risk that's involved. So if the score was out of twenty, and we can say, I'd like you to review this young man who I think may be suicidal and he's got a score of eighteen out of twenty on the (PRIMIS) score, then, again, I think that's a way of communicating between professionals so that everyone realises, because otherwise, that can be very frustrating about having to almost repeat your entire consultation that you've just spent sometimes twenty, thirty minutes or even more doing, you're pretty confident by that point that they are at risk, but it's then being able to convey that to other people...so I think that's why, in an increasingly, you know, combative world, that's why we quite like things that are very solid that we can rely on. (FG1 GPm1)

However, the majority of GPs reported serious concerns about the usefulness and acceptability of such a tool and its potential impact on the dynamics of a consultation. Concerns about its predictive validity and the possibility of its use leading to inappropriate referrals (false positives) or to high-risk cases not being correctly identified (false negatives) were also voiced. One GP who opposed to the use of screening tools raised concerns about assessing psychological problems in the same way as physical problems:

It's more difficult, though, isn't it, the psychological problems rather than physical where you say temperature is, blood pressure is...pulse is... (FG1 GPm4)

Instead, GPs supported the use of a guided decisionmaking tool that would facilitate a standardised way of recording risk history, ongoing social circumstances and informing clinical decision about management options.

Unless it [referring to risk assessment tool] was reliable, unless it actually actively...related to risk, you could then (underrate) people who were actually at risk, or overate loads of people. And it might be unhelpful...I mean, it's quite difficult, like, five questions more helpful than thirty years of GP experience, and likely to aid anything... (FG3 RF2)

\section{DISCUSSION}

In this study, we explored GPs' views and experiences of assessing, communicating with, and managing suicidal young people with the aim of co-producing an educational intervention on youth suicide prevention tailored to GPs' perceived needs.

The findings offer an original contribution to the extant literature in relation to the understanding and operationalisation of risk among GPs. Risk was perceived by some GPs as a discrete, categorical variable, the assessment of which should be based on validated screening tools in the same way risk for physical problems like sepsis or heart disease are assessed. Scores derived from such tools indicating severity levels could subsequently be used by GPs to justify their clinical decision. 'We live in an increasingly combative world' is what one GP said, which raises the question whether the use of screening tools is seen as a way of demonstrating that procedures have been followed and protecting one's clinical judgement. In contrast to this narrow understanding of risk, other GPs highlighted the multifactorial nature of risk that requires understanding the interplay between social, psychological and other ecological factors when conducting a risk assessment. These GPs supported the use of a guided decision-making tool that would allow them to conduct a holistic, structured, clinical assessment of suicide risk to determine future actions and treatment decisions. ${ }^{29} 30$

GPs' perceptions of young vulnerable people as impulsive, unpredictable and difficult to communicate with are confirmed by previous findings. ${ }^{31}{ }^{32}$ Rationalising self-harm as attention-seeking behaviour has been previously endorsed by health professionals, including GPs. ${ }^{33}$ These views could to some extent reflect GPs' frustration associated with organisational barriers, heavy workload and/or limited management options-'not knowing what to do with them' as one GP reported. However, it is also important to consider the extent to which these views could also result from how GPs conceptualise and understand risk. A narrowly focused identification of risk based on proximate, individual-level factors through the use of risk measurement tools or checklists would not adequately capture the emotional, psychological and social mechanisms that underlie self-harm and other risk factors. Distress, impulsivity, emotional pain and desperation are all key mechanisms associated with suicidal behaviours and self-harm 3435 and could easily be misinterpreted as attention-seeking behaviours and subsequently treated with negativity and lack of compassion. Such negative attitudes on behalf of clinicians have been shown to affect therapeutic engagement and management. $^{36}$

\section{Implications for practice}

This study has important implications for the provision of specialist training to support GPs in the assessment and management of youth suicide risk. Any such training should extend beyond the provision of microskills to enhancing GPs' competencies and capabilities in conducting a holistic, psychosocial needs-based assessment in line with National Institute for Health and Care Excellence (NICE) recommendations ${ }^{30}$ to facilitate therapeutic engagement and communication with young people. This training could potentially lead to early identification and intervention to address risk issues that might have otherwise gone undetected. ${ }^{37}$ An example of good practice is the HEADSS assessment (assessing the domains of Home, Education and employment, Activities, Drugs, Sexuality, Suicide/depression 
and Safety), ${ }^{38}$ used in primary care in Australia for assessing the psychosocial needs of young people and guiding clinical decision-making. The introduction of such an assessment in general practice in the UK would require a shift in GPs' conceptualisation of risk accompanied by changes in primary care organisation. This could include reviewing existing quality indicators, so that these are informed by national and international initiatives such as the WHO framework for the development of youth-friendly health services; ${ }^{39}$ promoting a collaborative model of care where young people are at the centre of the decisions made about their care. This would also require an improvement in the communication between GPs and young people to facilitate a trusting doctor-patient relationship and help bridge some of the barriers identified by the present study in relation to GPs' views of young people. We know that attitudes can influence clinical behaviour and practice ${ }^{40}$ but changing attitudes is a complex and multifaceted process, which is why the direct involvement of young people in the design and implementation of training would be vital in facilitating attitudinal change.

\section{Strengths and limitations}

The study was presented in line with COREQ $^{25}$ ensuring the comprehensive reporting of all study elements. Four focus groups and one in-depth interview were conducted and combined to enrich data and enhance depth of inquiry, increasing our understanding of the phenomenon under study. ${ }^{41}$ The data obtained from the in-depth interview revealed the same themes as the data obtained from the focus groups.

A limitation of this study is the use of convenience sampling based on accessibility, interest in the study and willingness to participate. Recruiting GPs with an interest in mental health might have led to a bias towards increased confidence in managing youth mental health issues including suicide risk. However, knowledge gaps and skill deficits in suicide risk assessment were still identified among those 'interested' GPs, supporting our argument for the provision of specialist education in this area.

Despite the richness of data collected, pragmatic constraints such as a time-limited space to conduct the focus groups might have influenced the depth of discussion and interaction among the participants on such a complex issue as youth suicide prevention.

\section{CONCLUSION}

The study revealed wide variations in the understanding and operationalisation of risk among GPs, which has subsequent implications to how GPs perceive risk should be assessed and their attitudes towards young suicidal people. GP education on suicide risk assessment and management in youth should extend beyond the provision of microskills to promoting a holistic understanding and assessment of risk and its individual, social and contextual influences.
Acknowledgements This work was supported by a NIHR Research Capability grant from Nottingham City Clinical Commissioning Group awarded to the corresponding author. The authors would like to thank the members of Project Steering Group who served as scientific advisors.

Contributors MM conceptualised the study. MM and LT designed the study. MM conducted all interviews and focus groups. LT and MM analysed the data. MM wrote the first draft of the manuscript. LT contributed to redrafting the manuscript. Both authors read and approved the final manuscript.

Funding This work was supported by a NIHR Research Capability grant from Nottingham City Clinical Commissioning Group awarded to MM.

Competing interests None declared.

Patient consent Obtained.

Ethics approval NRES Committee West Midlands-Coventry \& Warwickshire (14/WM/1100).

Provenance and peer review Not commissioned; externally peer reviewed.

Data sharing statement Additional data such as anonymised transcripts are available by emailing MM on maria.michail@nottingham.ac.uk.

Open Access This is an Open Access article distributed in accordance with the Creative Commons Attribution Non Commercial (CC BY-NC 4.0) license, which permits others to distribute, remix, adapt, build upon this work noncommercially, and license their derivative works on different terms, provided the original work is properly cited and the use is non-commercial. See: http:// creativecommons.org/licenses/by-nc/4.0/

\section{REFERENCES}

1. World Health Organization. Preventing suicide: a global imperative. Geneva, 2014.

2. Hawton K, Saunders KE, O'Connor RC. Self-harm and suicide in adolescents. Lancet 2012;379:2373-82.

3. Gould MS, Greenberg T, Velting DM, et al. Youth suicide risk and preventive interventions: a review of the past 10years. J Am Acad Child Adolesc Psychiatry 2003;42:386-405.

4. Welch SS. A review of the literature on the epidemiology of parasuicide in the general population. Psychiatr Serv 2001;52:368-75.

5. Hawton K, Houston K, Shepperd R. Suicide in young people. study of 174 cases, aged under 25 years, based on coroners' and medical records. Br J Psychiatry 1999;175:271-6.

6. Department of Health. Preventing suicide in England: one year on First annual report on the cross-government outcomes strategy to save lives, 2014.

7. Appleby L, Amos T, Doyle U, et al. General practitioners and young suicides. A preventive role for primary care. $\mathrm{Br} J$ Psychiatry 1996;168:330-3.

8. Luoma JB, Martin CE, Pearson JL. Contact with mental health and primary care providers before suicide: a review of the evidence. Am J Psychiatry 2002;159:909-16.

9. Goldberg P, Huxley P. Common mental disorders: a bio-social model. London: Rutledge, 1992.

10. Rickwood DJ, Deane FP, Wilson CJ. When and how do young people seek professional help for mental health problems? Med $J$ Aust 2007;187(7 Suppl):S35-9.

11. Mauerhofer A, Berchtold A, Michaud P, et al. GPs' role in the detection of psychological problems of young people: a population-based study. Br J Gen Pract 2009;59:e308-14.

12. Klein JD, McNulty M, Flatau CN. Adolescents' access to care: teenagers' self-reported use of services and perceived access to confidential care. Arch Pediatr Adolesc Med 1998;152:676-82.

13. Zimmer-Gembeck M, Alexander T, Nystrom RJ. Adolescents report their need for and use of health care services. J Adolesc Health 1997;21:388-99.

14. Royal College of Paediatrics \& Child Health. Bridging the gaps: health care for adolescents. London: RCPCH, 2003.

15. Saini $P$, Windfuhr $K$, Pearson $A$, et al. Suicide prevention in primary care: general practitioners' views on service availability. BMC Res Notes 2010;3:246-52.

16. Younes N, Chee CC, Turbelin C, et al. Particular difficulties faced by GPs with young adults who will attempt suicide: a cross-sectional study. BMC Fam Pract 2013;14:68. 
17. Taliaferro LA, Oberstar JV, Borowsky IW. Prevention of youth suicide: the role of the primary care physician. JCOM 2012;9:270-83.

18. Hider $\mathrm{P}$. Youth suicide prevention by primary healthcare professionals: a critical appraisal of the literature. New Zealand Health Technology Assessment (NZHTA), 1998

19. Gunnell DJ. The potential for preventing suicide. A review of the literature on the effectiveness of interventions aimed at preventing suicide. Bristol Health Care Unit: University of Bristol, 1994.

20. Fox F, Stallard P, Cooney G. GPs role identifying young people who selfharm: a mixed methods study. Fam Pract 2015;32:415-19.

21. Tait L. To disclose or not disclose psychological problems to GPs. Br J Gen Pract 2009;59:638-9.

22. World Health Organization. Public health action for the prevention of suicide: a framework. Geneva, 2012.

23. Mann JJ, Apter A, Bertolote J, et al. Suicide prevention strategies: a systematic review. JAMA 2005;294:2064-74.

24. Michail M, Tait L. Educational interventions for general practitioners to identify and manage depression as a suicide risk factor in young people: a systematic review and meta-analysis protocol. Syst Rev 2014;3:145.

25. Tong A, Sainsbury $P$, Craig J. Consolidated criteria for reporting qualitative research (COREQ): a 32-item checklist for interviews and focus groups. Int J Qual Health C 2007;19:349-57.

26. Ritchie J, Spencer L. Qualitative data analysis for applied policy research. In: Bryman A, Burgess RG, eds. Analysing qualitative data. London: Routledge, 1994:172-94.

27. Ritchie J, Spencer L, O'Connor W. Carrying out qualitative analysis In: Ritchie J, Lewis J, eds. Qualitative research practice: a guide for social science students and researchers. London: Sage Publications, 2003:219-62.

28. Ward DJ, Furber C, Tierney S, et al. Using Framework Analysis in nursing research: a worked example. J Adv Nurs 2013;69:2423-31.

29. Morriss R, Kapur N, Byng R. Assessing risk of suicide or self-harm in adults. BMJ 2013;347:f4572.
30. National Institute for Health and Clinical Excellence. Self-harm: longer-term management. (Clinical guideline CG133). 2012. http:// guidance.nice.org.uk/CG133

31. Illiffe S, Williams G, Fernandez V, et al. General practitioners' understanding of depression in young people: qualitative study. Prim Health Care Res Dev 2008;9:269-79.

32. Roberts $\mathrm{JH}$, Crosland A, Fulton J. "I think this is maybe our Achilles heel..." exploring GPs' responses to young people presenting with emotional distress in general practice: a qualitative study. BMJ Open 2013;3:e002927.

33. Herron J, Ticehurst $\mathrm{H}$, Appleby L, et al. Attitudes toward suicide prevention in front-line health staff. Suicide Life Threat Behav 2001;31:342-7.

34. Hawton K, Saunders KE, O'Connor RC. Series suicide 1 self-harm and suicide in adolescents. Lancet 2012;379:2373-82.

35. O'Connor RC, Rasmussen S, Hawton K. Distinguishing adolescents who think about self-harm from those who engage in self-harm. $\mathrm{Br} J$ Psychiatry 2012;200:330-5.

36. Thompson AR, Powis J, Carradice A. Community psychiatric nurses experience of working with people who engage in deliberate self-harm. Int J Ment Health Nurs 2008;17:153-61.

37. The Royal Australasian College of Physicians. Routine adolescent psychosocial health assessment. Position statement. The Royal Australasian College of Physicians, Sydney, 2008. Available from: http://www.racp.edu.au/fellows/resources/paediatric-resources

38. Goldenring JM, Rosen DS. Getting into adolescent heads: an essential update. Contemp Pediatr 2004;21:64.

39. Tylee A, Haller DM, Graham T, et al. Youth-friendly primary-care services: how are we doing and what more needs to be done? Lancet 2007;369:1565-73.

40. Saunders KE, Hawton K, Fortune S, et al. Attitudes and knowledge of clinical staff regarding people who self-harm: a systematic review. $\checkmark$ Affect Disord 2012;139:205-16.

41. Lambert SD, Loiselle CG. Combining individual interviews and focus groups to enhance data richness. J Adv Nurs 2008;62:228-37. 